\title{
Über die Einwirkung von Kohlenoxychlorid auf Aluminiumhaloide.
}

\author{
I. Mitteilung. \\ Von
}

A. VON Bartal.

Die Einwirkung von Phosgen auf Aluminiumchlorid wurde von E. BAUD untersucht. ${ }^{1}$ Er löste Aluminiumchlorid in flüssigem Phosgen auf und exhielt auf diese Weise mehrere Doppelrerbindungen von der Zusammensetzung: $2 \mathrm{AlCl}_{3} .5 \mathrm{COCl}_{2} ; 2 \mathrm{AlCl}_{3} .3 \mathrm{COCl}_{2} ; 4 \mathrm{AlCl}_{3}$. $\mathrm{COCl}_{2}$ usw.

Die Einwirkung auf Aluminiumbromid wurde, allerdings nur ganz oberflächlich von Besson studiert, ${ }^{2}$ als er nach einer Darstellungsmethode für Koblenoxybromid suchte. Nach seinen Angaben ${ }^{3}$ entstehen auch hierbei Doppelverbindungen, jedoch keine Kohlenoxybromide. Genauer hat er die entstandenen Produkte nicht untersucht.

$\mathrm{Da}$ in dieser Richtung hin weitere Veröffentlichungen nicht erschienen sind, stellte ich mir die Aufgabe, die Einwirkung von Kohlenoxychlorid auf Aluminiumbromid, Aluminiumjodid und Aluminiumfluorid genau zu untersuchen. Im folgenden möchte ich vorläufig die Resultate der Versuche mit Aluminiumbromid veröffentlichen, welche zum Abschlufs gebracht werden konnten, über die Einwirkung auf Aluminiumjodid gedenke ich in kurzem ebenfalls Mitteilung machen zu können.

Einwirkung von Kohlenoxychlorid auf Aluminiumbromid.

Das zu den nachstehenden Versuchen verwendete Aluminiumbromid wurde nach G. Gustavson dargestellt, ${ }^{4}$ indem über Aluminium-

1 Compt. rend. 140, 1688-89.

Compt. rend. 120, 190-92.

${ }^{3}$ Compt. rend. 120, 190-92.

4 Journ. pralit. Chem. [2] 63, 110-112. 
blechstücke, welche sich in einer Röhre befanden, Bromdampf geleitet wurde. Das erhaltene, von den nicht angegriffenen Aluminiumstücken sorgfältig getrennte Produkt ist von anhaftendem Brom braun gefärbt. Eine Reinigung durch Sublimation erwies sich jedoch als nicht notwendig, deshalb wurde dieses Produkt ohne weiteres zu den Versuchen verwendet.

Es zeigte sich nun, dafs der Gang der Reaktion ein verschiedener ist, je nachdem das Aluminiumbromid, oder aber das Kohlenoxychlorid während der Reaktion im Überschufs vorhanden ist.

\section{a) Aluminiumbromid im Überschufs.}

$100 \mathrm{~g}$ Aluminiumbromid wurden in einem Fraktionierkolben im lebhaft siedenden Wasserbade zum Schmelzen gebracht und in die Flüssigkeit Phosgen, welches vorher eine Waschflasche mit Schwefelsäure passierte, in raschem Strome eingeleitet. Die Ableitungsröhre des Kolbens stand mit einem zweiten Fraktionierkolben in Verbindung, welcher mit einer Kältemischung von Eiskochsalz gekühlt war. Die Reaktion geht glatt von statten; in der gekühlten Vorlage sammelt sich eine braunrote Flüssigkeit an. Nach und nach wird der Inhalt des Siedegefälses vollständig fest. Sobald flüssige Anteile nicht mehr vorhanden sind, unterbricht man den Phosgenstrom, ${ }^{1}$ stellt das Wasserbad beiseite und lälst den Kolbeninhalt erkalten.

Das in der gekühlten Vorlage verdichtete Destillat ist dunkelrot und raucht an der Luft. Es destilliert fast konstant bei $\mathbf{5 8}$ bis $60^{\circ}$ über und hinterlälst ein wenig festen Rückstand, welcher aus mit hinübergerissenem Aluminiumbromid besteht. Das Destillat enthält zum gröIsten Teile freies Brom, daneben ganz wenig Kohlenoxybromid, welches aus gröfseren Mengen des Destillates, durch Schütteln mit Quecksilber bis zum Farbloswerden und abdestillieren, auch rein erhalten werden kann. Näheres über die Reindarstellung und Eigenschaften dieses Körpers in meiner Abhandlung: „Über das Kohlenoxybromid". ${ }^{2}$

Der Inhalt des Siedegefälses ist eine rotbraune, strahlig-krystal-

${ }_{1}$ Um mit kleineren Mengen Phosgen bequem und unbelästigt durch seinen Geruch experimentieren zu können, benutzt man zweckmälsig die von mir konstruierte „Einschmelzflasche mit Hahn für leicht verdichtbare Gase“, $C h . Z$. 39 (1907).

2 Lieb. Ann. 345, 335-53. 
linische Masse, welche an der Luft raucht und zerfliefst; sie enthält noch etwas freies Brom und riecht ein wenig nach Phosgen.

Die eine Hälfte dieses Reaktionsproduktes wurde im Vakuum der Sublimation unterworfen. ${ }^{1}$ Hierbei entweicht zuerst das anhaftende Brom, dann bei $110-120^{\circ}$ sublimiert der grölste Teil der Substanz in kleinen, ziegelroten Blättchen über. Ein wenig Rückstand bleibt als grauweifse Masse zurück, welche auch beim Glühen nicht sublimiert und nicht schmilzt; sie besteht aus Aluminiumoxyd, dessen Bildung von einer geringen Zersetzung herrührt. Das Sublimat wurde zur Analyse gebracht: ${ }^{2}$

0.5825 g Subst.: $1.5462 \mathrm{AgCl}+\mathrm{AgBr} ; 0.6092 \mathrm{AgBr}, 0.9370 \mathrm{AgCl}$; $0.1635 \mathrm{Al}_{2} \mathrm{O}_{3}$.

Gefunden . . . . . . Br: 44.5, Cl: 39.8, Al: 14.9 .

Berechnet für $\mathrm{AlCl}_{2} \mathrm{Br}=176.6: \quad, \quad 45.0, \quad$, 39.8, , 15.2 .

Das Aluminiumchlorobromid stellt hellziegelrote, kleine Krystallblättchen dar, welche an der Luft rauchen und zerfliefsen. Es löst sich in Wasser unter Wärmeentwickelung, jedoch nicht so stürmisch wie Aluminiumbromid zu einer schwachgelblichen, klaren Flüssigkeit auf. In einem Vakuum von $15-20 \mathrm{~mm}$ sublimiert es fast unzersetzt bei $110-120^{\circ}$, unter gewöhnlichem Drucke bei $130-135^{\circ}$, wobei aber schon eine teilweise Zersetzung stattfindet. Im offenen Rohre schmilzt es nicht, sondern sublimiert unter Zersetzung; rasch auf Rotglut erhitzt, zersetzt es sich beinahe voll. ständig in Aluminiumoxyd, Chlor und Brom.

Im zugeschmolzenen Röhrchen schmilzt es bei $142-143^{\circ}$.

Das Aluminiumchlorobromid verhält sich also ähnlich wie Aluminiumchlorid, d. h. sein Schmelzpunkt liegt höher als sein Sublimationspunkt.

Die zweite Hälfte des Reaktionsproduktes wurde in viel Wasser unter Eiskühlung gelöst, wodurch eine schmutzigbräunliche, trübe Flüssigkeit entstand. Beim Erhitzen bis zum Sieden klärt sich diese, indem sich ganz geringe Mengen eines dunkelbraunen, flockigen Niederschlages abscheiden. Die fast farblose, klare Lösung wurde

${ }^{1}$ Die Sublimation wurde in dem Apparat für Vakuumsublimation von R. KемpF, Ch. $Z .100$ (1906), ausgeführt.

${ }^{2}$ Das Auflösen geschah direkt in einer mit Salpetersäure angesäuerten, stark verdünnten Silbernitratlösung; zur Kontrolle wurde in einer zweiten Probe die Halogenbestimmung im zugeschmolzenen Rohre nach CARIUs ausgeführt, welche mit dem ersteren gut übereinstimmende Resultate lieferte. 
abfiltriert und der braune Rückstand nach dem Auswaschen mit Wasser bei $100^{\circ}$ getrocknet. Von der in trockenem Zustande schwarzbraunen Substanz wurde jedoch so wenig erhalten, dafs sie weder vom Filter getrennt, noch zur Analyse gebracht werden konnte. Die klare Lösung wurde siedend eingeengt, dann auf dem Wasserbade bei $70-80^{\circ}$ sehr vorsichtig, zuletzt unter Umrühren mit einem Glasstabe, vollends eingedampft. So resultiert ein hellrosenrotes bis gelbliches Krystallpulver, dessen Volumen etwa das anderhalbfache, bis doppelte der ursprünglichen Substanz beträgt. Die Analyse ergab folgende Werte:

0.6290 g Subst.: $1.0236 \mathrm{AgBr}+\mathrm{AgCl} ; 0.4035 \mathrm{AgBr}, 0.6201 \mathrm{AgCl}$; $0.1125 \mathrm{Al}_{2} \mathrm{O}_{3}$.

Gefunden . . . . . . . . . Br: 27.3, Cl:24.4, Al: 9.5 .

Berechn. für $\mathrm{AlCl}_{2} \mathrm{Br}+6 \mathrm{H}_{2} \mathrm{O}=283.9$ : , 27.9, „24.7, „9.4.

Das Aluminiumchlorobromid-Hexahydrat bildet schwachgefärbte, mikroskopische Krystalle, welche an Glas ein wenig haften; sie rauchen nicht an der Luft, ziehen jedoch langsam Wasser an und zerfliefsen nach einiger Zeit. Die Verbindung schmilzt weder im offenen, noch im zugeschmolzenen Rohre, verbält sich also genau wie das $\mathrm{AlBr}_{3}+6 \mathrm{H}_{2} \mathrm{O}$; beim starken Erhitzen gibt sie unter Zersetzung Wasser ab. Sie ist in Wasser ohne merkliche Wärmeentwickelung klar löslich zu einer schwachgelblichen Flüssigkeit, welche beim vorsichtigen Eindampfen wieder das Hexahydrat liefert.

Wird das sublimierte Aluminiumchlorobromid in Wasser gelöst und die Lösung eingedampft, so erhält man ebenfalls das Hexahydrat.

Auf Grund obiger Beobachtungen muls die Reaktion im Sinne nachstehender Gleichung verlaufen:

$$
\mathrm{AlBr}_{3}+\mathrm{COCl}_{2}=\mathrm{AlCl}_{2} \mathrm{Br}+\mathrm{CO}+\mathrm{Br}_{2} .
$$

In statu nascendi bildet sich wahrscheinlich hauptsächlich Kohlenoxybromid, welches aber bei $100^{\circ}$ wieder grölstenteils in Kohlenoxyd und Brom zersetzt wird; deshalb enthält das Destillat nur ganz geringe Mengen dieses Körpers. Die Ausbeute an reinem Aluminiumchlorobromid beträgt ca. $80 \%$ der Theorie; die Menge des verbrauchten Phosgens entspricht fast genau der berechneten. Die Reaktion verläuft also nahezu quantitativ.

b) Kohlenoxychlorid im Überschufs.

In einem Rundkolben von $250 \mathrm{ccm}$ Inhalt und etwa $30 \mathrm{~cm}$ Halslänge wurde unter Anwendung einer Kältemischung $25 \mathrm{~g}$ flüssiges 
Kohlenoxychlorid hineingebracht. Der Kolben ist mit einem doppelt durchbohrten Korke verschlossen, in dessen eine Bohrung eine dünne, kurze, beiderseits offene Glasröhre als Ableitungsröhre, in dessen andere Bohrung ein weites, $30 \mathrm{~cm}$ langes Rohr befestigt wird; dieses weite Rohr ist mit Hilfe eines $10 \mathrm{~cm}$ langen, guten Kautschukschlauches mit einem Kölbchen verbunden, welches $25 \mathrm{~g}$ fein pulverisiertes Aluminiumbromid enthält. Um während der Reaktion die Kugel des Kolbens nicht kühlen, und trotzdem kein Koblenoxychlorid verlieren zu müssen, wurde der Kolbenhals in seiner ganzen Länge mit einer Kältemischung von Eis und Kochsalz umgeben. Hierzu benutzt man zweckmäfsig ein grölseres, hohes Präparatenglas mit abgesprengtem Boden, welches man durch Vermittelung eines durchbohrten Korkes dicht oberhalb der Kolbenkugel an dessen Halse befestigt und mit der Kältemischung füllt.

Nun wurde das Aluminiumbromid, durch vorsichtiges Neigen und Schütteln des Kölbchens, in kleinen Portionen nach und nach zu dem Phosgen gegeben. Die Reaktion ist ziemlich lebhaft; das Aluminiumbromid löst sich in dem Phosgen auf, die Flüssigkeit gerät ins Sieden und färbt sich braun. In kurzer Zeit ist die Reaktion beendet, indem das Reaktionsprodukt zu sieden aufhört. Es wurde in einen Fraktionierkolben gegossen (ein fester Rückstand ist nicht vorhanden) und am Wasserbade destilliert. Bei $15^{\circ}$ tritt plötzlich eine Zersetzung ein: unter stürmischem Aufbrausen ging Kohlenoxychlorid über, das durch etwas Brom gefärbt war, dann stieg die Quecksilbersäule langsam bis $56^{\circ}$, während eine rotgelbe, durchsichtige Flüssigkeit überdestillierte. Im Siedegefäfs blieben gröfsere Mengen einer gelblichen Masse zurück, welche sich als Aluminiumchlorid (durch etwas Brom verunreinigt) erwies.

Das Destillat wurde im Wasserbade fraktioniert und die Fraktionen bis $20^{\circ}$, von $20-45^{\circ}$ und von $45^{\circ}$ bis etwa $59^{\circ}$ gesondert aufgefangen; der grölste Teil destilliert bei $20-45^{\circ}$ über. Die erste Fraktion (bis $20^{\circ}$ ) bestand grölstenteils aus unverändertem, durch Brom gefärbtem Kohlenoxychlorid; die dritte Fraktion (45 bis $\left.59^{\circ}\right)$ aus Brom. Die zweite Fraktion $\left(20-45^{\circ}\right.$ ) enthielt ebenfalls sehr viel Brom, wurde aber beim Schütteln mit Quecksilber allmählich heller, bis schliefslich 2-3 ccm einer farblosen Flüssigkeit zurückblieben, welche bei etwa $25^{\circ}$ siedete. Dieses niedrigsiedende Reaktionsprodukt erwies sich als das bis jetzt nicht in reinem Zustande erhaltene Kohlenoxychlorobromid. 
c) Darstellung von Kohlenoxychlorobromid COClBr.

Besson $^{1}$ will Kohlenoxychlorobromid (neben Kohlenoxybromid) bereits durch Einwirkenlassen von Borbromid auf Phosgen unter Druck erhalten und in reinem Zustande als eine bei $36-38^{\circ}$ siedende Flüssigkeit isoliert haben. BROCHET ${ }^{2}$ konnte aber nach diesem Verfahren weder das Kohlenoxybromid, noch das Chlorobromid erhalten und ich machte die gleiche Erfahrung. ${ }^{3}$ Aus dem Nachstehenden ist ersichtlich, daís das reine Kohlenoxychlorobromid einen erheblich niedrigeren Siedepunkt hat wie ihn Besson bei seiner Verbindung fand, wodurch abermals bestätigt wird, dafs der Körper, den Besson für das Chlorobromid hielt, nicht die reine Verbindung, sondern im besten Falle nur ein Gemenge dieser mit höhersiedenden Bestandteilen war.

$\mathrm{Zu}$ der Darstellung nimmt man die Ausgangsmaterialien in demselben Mengenverhältnis wie oben angegeben; man gibt in eine mit Eiskochsalzmischung gekühlte Bombenröhre $25 \mathrm{~g}$ Phosgen und dann nach und wach $25 \mathrm{~g}$ fein pulverisiertes Aluminiumbromid. Nachdem sich alles gelöst und die anfangs ziemlich lebhafte Reaktion nachgelassen hat, schmilzt man die Röhre zu und erhitzt $7-8$ Stunden auf $140-145^{\circ}$. Aus dem Ofen herausgenommen, bildet das Reaktionsprodukt eine rotbraune, helldurehsichtige Flüssigkeit, welche in eine Kältemischung gestellt, erstarrt und herausgenommen bei etwa $2-3^{0}$ schmilzt. Das Reaktionsprodukt wird aus einem Fraktionierkolben am Wasserbade abdestilliert, wobei der flüssige Bestandteil bei $40-42^{\circ}$ vollständig übergeht, während im Siedegefäfs Aluminiumchlorid zurückbleibt. Das Destillat wird fraktioniert und die bei $20-40^{\circ}$ übergehenden Anteile gesondert aufgefangen. Sie bilden eine rote, durchsichtige, leichtbewegliche Flüssigkeit, welche mit Quecksilber geschüttelt, sich allmählich entfärbt. Die farblose Flüssigkeit destilliert bei $22-25^{\circ}$ und enthält noch Spuren von Phosgen. Um auch diese Spuren vollständig zu entfernen, fraktioniert man das Produkt noch einmal und fängt die bei $24.5-25.5^{\circ}$ übergehenden Anteile auf.

Die Analyse dieses Produktes ergab folgende Werte:

$0.4465 \mathrm{~g}$ Substanz: $1.0232 \mathrm{AgCl}+\mathrm{AgBr}=0.8859 \mathrm{AgCl}$; $0.5795 \mathrm{AgBr}, 0.4737 \mathrm{AgCl}$.

1 Compt. rend. 120, 190-92.

B Bull. soc. chim. [3] 17, 224.

${ }^{3}$ Lieb. Ann. 345, 335-53. 
$0.5030 \mathrm{~g}$ Substanz: $0.1522 \mathrm{~g} \mathrm{CO}_{2}$, Spuren von $\mathrm{H}_{2} \mathrm{O}$.

Gefunden . . . . . . . Br: 55.2, Cl: 24.5, C: 8.2.

Berechnet für $\mathrm{COClBr}=142.3: \quad, \quad 55.7 \quad$, 24.7, „, 8.3 .

Bestimmung der Damptdichte:

0.4535 g Substanz: $79.6 \mathrm{ccm}$ bei $22^{\circ}$ und $758 \mathrm{~mm}$.

Gefunden: 4.91. Berechnet für $\mathrm{COClBr}=142.3: 4.94$.

Das Kohlenoxychlorobromid ist eine farblose, bis schwach gelbliche, leicht bewegliche Flüssigkeit, mit einem äufserst unangenehmen, scharfen Phosgengeruch. An der Luft raucht es nicht, zieht jedoch Wasser an und trübt sich; am Sonnenlicht färbt es sich gelblich. Die reine Verbindung siedet bei $25^{\circ}$ unzersetzt und hat bei $15^{\circ}$ das spezifische Gewicht 1.82. Im allgemeinen besitzt es ganz ähnliche Eigenschaften wie Kohlenoxychlorid und Kohlenoxybromid, ist aber beständiger als letzteres. Bei $100^{\circ}$ zersetzt es sich noch nicht und wird von Quecksilber selbst bei Siedehitze nicht angegriffen; Antimon hingegen wirkt auf ihn ein. Mit Dimethylanilin reagiert es stürmisch unter Bildung von Krystallviolett.

Ist also während der Reaktion Phosgen im Überschufs vorhanden, so nimmt diese den folgenden Gang:

$$
\mathrm{AlBr}_{3}+3 \mathrm{COCl}_{2}=\mathrm{AlCl}_{3}+3 \mathrm{COClBr} \text {. }
$$

Zuerst bildet sich hierbei bestimmt eine Doppelverbindung, entweder von Aluminiumbromid mit Phosgen, oder Aluminiumchlorid mit Phosgen bzw. Kohlenoxychlorobromid, welches sich aber beim Destillieren des Reaktionsgemisches bei $15^{\circ}$ wieder zersetzt. $\mathrm{Da}$ das Kohlenoxychlorobromid beständiger ist als das Kohlenoxybromid, zerfällt es nicht wieder und kann daher aus dem Reaktionsprodukt gewonnen werden. Die Ausbeute an reinem Kohlenoxychlorobromid beträgt bis $50 \%$ der Theorie.

Berlin, Anorgan. Laboratorium der Technischen Hochschule.

Bei der Redaktion eingegangen am 9. Juli 1907. 